REFERENCIA: Rigo, Y. D. (2021). Percepciones de estudiantes sobre compromiso académico y clima del aula. Tendencias pasadas, presentes y futuras sobre una educación superior en transición. ENSAYOS, Revista de la Facultad de Educación de Albacete, 36(2), 77-96. Enlace web: http://www.revista.uclm.es/index.php/ensayos - Consultada en fecha (dd-mm-aaaa)

\title{
PERCEPCIONES DE ESTUDIANTES SOBRE COMPROMISO ACADÉMICO Y CLIMA DEL AULA. TENDENCIAS PASADAS, PRESENTES Y FUTURAS SOBRE UNA EDUCACIÓN SUPERIOR EN TRANSICIÓN
}

\section{STUDENTS' PERCEPTIONS ON ACADEMIC ENGAGEMENT AND CLASSROOM CLIMATE. PAST, PRESENT AND FUTURE TRENDS IN HIGHER EDUCATION IN TRANSITION}

\author{
Daiana Yamila Rigo \\ dyrigo@hum.unrc.edu.ar \\ Instituto de Investigaciones Sociales, Territoriales y Educativas (ISTE) \\ Consejo Nacional de Investigaciones científicas y Técnicas (CONICET) \\ Universidad Nacional de Río Cuarto (Argentina)
}

Recibido: $23 / 12 / 2020$

Aceptado: 17/11/2021

\begin{abstract}
Resumen:
El artículo presenta dos estudios, uno desarrollado en el año 2019 y otro en el 2020, que tuvieron como objetivo general describir la percepción de estudiantes de nivel superior de educación sobre el clima de clase -presencial y virtual- y el compromiso académico antes y durante la pandemia. En total participaron 211 estudiantes argentinos, 102 del primer estudio con una edad media de 20,55 años ( $S D=3,7)$, y 109 del segundo estudio, con un promedio de 22,3 años $(S D=4,1)$. La investigación se desarrolló bajo una metodología mixta, utilizando para la recolección de datos tres instrumentos, dos estandarizados, para valorar clima de la clase y compromiso académico, y el otro de preguntas abiertas. Los resultados encontrados, en términos generales, mostraron diferencias estadísticamente significativas en la comparación por año, especialmente en todas las dimensiones del compromiso académico y del clima del aula, a excepción de equipamiento, a favor de la educación en pandemia. Asimismo, dan cuenta de la importancia de expandir los contextos educativos, promover la agencia y repensar la evaluación. Concluyendo sobre la importancia de pensar una educación en transición que valore aspectos positivos de la presencialidad y la virtualidad.
\end{abstract}

Palabras clave: agencia; educación expandida; compromiso; clima del aula.

\footnotetext{
Abstract:

The article presents two studies, one developed in 2019 and the other in 2020, which had the general objective of describing the perception of students of higher education level about the classroom climate - face-to-face and virtual - and the academic engagement before and during the pandemic. In total, 211 Argentine students participated, 102 from the first study with a mean
} 
age of 20.55 years $(S D=3.7)$, and 109 from the second study, with an average age of 22.3 years $(S D=4.1)$. The research was developed under a mixed methodology, using for data collection three instruments, two standardized, to assess class climate and academic engagement, and the other of open questions. The results found, in general terms, showed statistically significant differences in the comparison by year, especially in all dimensions of academic engagement and classroom climate, except for equipment, in favor of education in pandemic. They also realize the importance of expanding educational contexts, promote the agency and rethinking evaluation. Concluding on the importance of thinking about an education in transition that values positive aspects of face-to-face and virtuality.

Keywords: agency; expanded education; engagement; classroom climate.

\section{Introducción}

Se inicia esta investigación con un interrogante que muchos investigadores, docentes y estudiantes tienen en mente y hace eco en los escenarios educativos actuales, ¿lo evidenciado hoy en educación, en términos de virtualidad, es quizás la crónica de una revolución anunciada? Se podrían optar, al menos, por tres respuestas, una clara negación, una afirmación tajante, o una transición necesaria en virtud de los cambios y avances en los modos de acceder a la información, construir el conocimiento y debatir en red.

La convivencia, de lo presencial y lo virtual, es innegable. Sangrá Morer (2006) coincide en que las prácticas de educación a distancia (EaD) desembarcaron con el objetivo de hacer llegar la educación a todo aquel que la necesitaba. Rama (2008, p. 42) puntualiza que "la EaD no constituye una modalidad educativa estática, sino que se presenta en una diversidad de opciones, entre las que destacan la semipresencial, la virtual y la a distancia, con múltiples combinaciones y mezclas híbridas". Heedy y Uribe (2008), plantean que la EaD es tanto una modalidad como una estrategia educativa que permite que los elementos de espacio y tiempo, no determinen los procesos de enseñanza y de aprendizaje.

A nivel mundial, las universidades a distancia hicieron su aparición en los años 70 del siglo XX, como respuesta a la nueva competencia y la evaluación del mercado de las nuevas tecnologías de información y comunicación como el resultado del desarrollo de la propia EaD en los diversos países. Las primeras experiencias tuvieron un amplio apoyo y asociación con instituciones internacionales, que expresaban la presencia de la nueva educación trasnacional en la región (Rama, 2008).

Específicamente, Fanelli, Marquina y Rabossi (2020) marcan al siglo XXI como la expansión de la educación virtual universitaria de manera notable en Argentina. Durante el 2017, el sistema universitario comenzó a expandirse de la mano de un acuerdo legal sobre condiciones institucionales que pudieran asegurar la calidad de la modalidad virtual, a través de diversos procesos de acreditación. Las instituciones dispuestas a ofrecer instancias educativas a distancia, total o parcial, debieron crear un Sistema Institucional de Educación a Distancia propio, valorado tanto por la SPU - Secretaría de Políticas Universitarias-, como por la CONEAU -Comisión Nacional de Evaluación y Acreditación Universitaria-. En consonancia con el avance de la educación virtual, Chiecher y Donolo (2006) advierte la reimpresión de algunas dificultades, problemas e interrogantes sobre la enseñanza y el aprendizaje que ya habían sido estudiados en contextos de presencialidad.

Lo cierto es que, en el estado de avance de la educación virtual, junto a las dificultades compartidas, a inicios del 2020 la educación muta de escenario como respuesta sanitaria al 
COVID-19. Los intentos de virtualización de la educación en las instituciones educativas de nivel superior se caracterizan por la diversidad (Cimadevilla, 2020). Tal como lo postula Ordorika (2020, p. 1), los actores sociales que conforman la educación superior "han tenido que hacer frente con premura y creatividad al reto de reorganizar sus actividades para dar continuidad al ejercicio de sus funciones sustantivas y para seguir atendiendo los retos y problemas que enfrentaban desde antes de que estallara la crisis del COVID-19". Ello amerita escuchar voces y describir las percepciones de los agentes educativos para comprender esa transición de entornos de cara al futuro educativo. En particular en esta investigación, las construidas por los estudiantes de nivel superior.

Se presenta una investigación que reúne los dos escenarios de la transición, las clases presenciales por un lado y las virtuales por otro, para repensar la educación postpandemia. Concretamente, se desarrollaron dos estudios, uno en el 2019 y otro en el 2020, que tuvieron como objetivo describir cómo los estudiantes se comprometen y perciben el clima de la clase, en contextos académicos presenciales y virtuales. Asimismo, se buscó indagar sobre las actividades de aprendizaje más relevantes, ya sea por su modalidad, contenido o contexto en la cual se desarrolló, junto a las dimensiones del clima de la clase que contribuyeron o, no a sus intereses, participación, autorregulación y expresión de perspectivas.

Se considera importante estudiar ambos escenarios, como modo de reconocer e identificar actividades y contextos de aprendizaje que permitan definir tendencias futuras a base de las pasadas y las presentes, a modo de rescatar aquellos elementos de clima del aula que promuevan el compromiso académico de los estudiantes que se encuentran en el nivel superior de educación. Una temática que repetidamente se vincula con problemáticas que aún movilizan nuevas indagaciones, como la desvinculación, deserción y ralentización (Rigo y Donolo, 2019).

\section{Compromiso académico}

Los aportes más recientes sobre el compromiso académico expresan que además de tratarse de un metaconstructo, es a la vez dinámico, situado y modificable (Kahu, 2013; Zhang y McNamara, 2018). En consonancia, los desarrollos sobre el clima de aula lo definen también como una construcción compleja y maleable (Dreesman, 1982). Por lo que se podría esperar que la percepción de los estudiantes sobre el compromiso académico desarrollado y el clima de aula apreciado estén mediados por la modalidad educativa en el que el proceso de aprendizaje se desarrolla, es decir, presencial o virtual. Considerándose que cada una de estas modalidades podría implicar distintas percepciones sobre el clima del aula y los componentes cognitivos, conductuales, afectivos y agénticos del compromiso los estudiantes.

El compromiso académico de los estudiantes es un término definido desde múltiples perspectivas teorías (Kuh, Kinzie, Buckley, Bridges y Hayek, 2007; Trowler, 2010; Vibert y Shields, 2003), con algunos puntos de acuerdo, que convergen en la idea de una participación efectiva en prácticas educativas diversas. Concretamente, Harrison (2014) ahonda en la perspectiva holística que integra la comportamental, psicológica y socio-cultural, para entender al compromiso como un proceso de co-creación, que abarca numerosas características descriptas por Kahu (2013).

La primera característica alude a una experiencia interactiva, en tanto el compromiso refleja un estado psicológico en respuesta al intercambio sostenido con un objeto determinado, como las tareas de aprendizaje. La segunda refiere a su carácter dinámico e iterativo, es decir, el proceso de participación de los estudiantes puede pensarse como una serie de estados que responden al contexto interactivo, lo que lo hace maleable. Asimismo, se lo describe como un proceso social, en tanto el compromiso de los estudiantes no opera de forma aislada, sino integrado en 
una red más amplia de relaciones e interacciones. Intercambios que no se limitan al aula o la clase, sino que están influenciados por condiciones situacionales más amplias, curriculares, institucionales y territoriales. Por último, representa un meta-constructo, integrado por tres dimensiones: conductual, afectiva y cognitiva (Harrison, 2014; Kahu, 2013) y, una nueva dimensión más reciente, la agéntica, para argumentar que los estudiantes se involucran en las actividades de aprendizaje que proporcionan sus profesores, y que, además, lo hacen para comunicar sus preferencias, ofrecer opiniones sobre la clase y hacer preguntas para seguir aprendiendo (Reeve, Cheon y Jang, 2020).

La cuarta dimensión del compromiso académico, refiere a las iniciativas que toman los estudiantes para moldear el contexto instructivo, y contribuir al fluir de la clase en vista a mejorar sus condiciones de aprendizaje. El compromiso agéntico, plantean Reeve (2013), Reeve y Shin (2020) y Rigo (2020) son los actos intencionales, proactivos y constructivos que los estudiantes despliegan para mejorar sus contextos y oportunidades de aprendizaje, enriqueciendo la experiencia educativa. Se define en torno a tres rasgos, proactivo, al dar lugar a la voz de las motivaciones internas de los estudiantes de cara a moldear el contexto de enseñanza; constructivo, en el sentido de que los actos de participación de los agentes son iniciativas para progresar y conseguir un mayor apoyo de los profesores; y recíproco, en tanto el estudiante trabaja en colaboración con los docentes para trasformar el contexto de la clase.

Por el contrario, las otras tres dimensiones del compromiso son reactivas al contexto instructivo configurado por el docente (Reeve, 2013). Es decir, Fredricks, Blumenfeld y Paris (2004) proponen que las dimensiones, afectiva, cognitiva y conductual, resultan de la interacción de los sujetos con el contexto y responden a las variaciones de este. Específicamente, el contexto de la clase y su clima es considerado por Arguedas Negrini (2010) y González González (2010) como un factor crítico que afecta al involucramiento. Son las percepciones que los estudiantes construyen sobre los apoyos recibidos pueden moldear la implicación académica. Shernoff (2013) postula que las dimensiones contextuales tienen un mayor peso, en relación con los personales, en la expresión de las manifestaciones cognitivas, conductuales y afectivas de los estudiantes, y Rigo (2020) agrega que también sobre las posibilidades de expresar sus agencias.

\section{Clima de la clase}

El clima o ambiente de la clase (Fernández-Ballesteros y Sierra, 1989; Wheldall, Beaman y Mok, 1999), ha cobrado un interés creciente en la calidad del entorno del aula, en las últimas décadas. Relacionado significativamente con mejores progresos a nivel académico y afectivo de los estudiantes (Galini y Efthymia, 2009).

Con diversidad de definiciones, el clima de clase es una combinación de las percepciones de profesores y estudiantes durante un encuentro de aprendizaje como resultado del comportamiento del profesor y el comportamiento de los estudiantes mientras se realizan actividades de aprendizaje. La construcción del clima de las aulas es un proceso continuo. Al inicio de una actividad, el profesor desencadena el clima del aula. Más tarde, el profesor controla el clima del aula promoviendo el comportamiento de los estudiantes. Después, los estudiantes contribuyen al clima del aula para que el aprendizaje tenga lugar (Sriklaub, Wongwanich y Wiratchai, 2015).

Es un proceso que Dreesman (1982) caracteriza como dinámico y social, es decir, que contempla las percepciones de los estudiantes en el intercambio activo del grupo aula. Pereira (2010) sostiene que el clima que se genera en las aulas está determinado, en gran parte, por los comportamientos de docentes. Pianta, La Paro y Hamre (2008) argumentan que las 
motivaciones internas de los estudiantes también median las percepciones que los estudiantes construyen sobre el clima del aula.

En ese interjuego de factores internos y externos, el clima de la clase es una co-construcción multidimensional. Ríos, Bozzo, Marchant y Fernández (2010) entienden que se compone de una parte material (mobiliario e infraestructura edilicia) y otra inmaterial (sujetos, relaciones interpersonales, contendido, metodología y evaluaciones). Estudios recientes en contextos educativos virtuales las redefinen para precisar que lo material hace a las conexiones a internet, espacios físicos en el hogar y equipos tecnológicos disponibles, mientras que lo inmaterial si bien refieren a las personas, los intercambios, las actividades, los recursos didácticos y evaluaciones, están tienen lugar desde plataformas virtuales, Google Meet, Zoom o Jitsi Meet, asumiendo características particulares en cuanto relaciones sincrónicas y asincrónicas desde mediaciones tecnológicas diversas (Rigo, 2020).

El compromiso y los modos de configurar el clima de la clase en función entornos presenciales o virtuales tienen algunos antecedentes pre-pandemia que muestran algunas tendencias, que se pretenden profundizar con el estudio presentado. Ivala y Gachago (2012), destacan en su estudio el potencial de Facebook y los blogs para mejorar la participación de los estudiantes en el aprendizaje tanto dentro como fuera del contexto de los contextos académicos. El artículo sugiere que las tecnologías en la vida diaria de los estudiantes, como Facebook, debe utilizarse en la educación superior para promover la interacción de los estudiantes y un mayor compromiso con los materiales de aprendizaje.

Deng, Benckendorff y Gannaway (2020) concluyen que las características de los alumnos que aprenden por MOOC - Massive Open Online Course- y quienes lo hacen en el campus universitario pueden ser muy diferentes, pudiéndose capturar patrones de participación y compromiso que son idiosincrásicos a cada grupo. Concretamente, Suárez Riveiro y Anaya Nieto (2004) enfatizan que la modalidad educativa en la que el proceso de aprendizaje se desarrolla, presencial o a distancia, implica distintas influencias sobre el proceso de aprendizaje desarrollado por los estudiantes, y sus componentes cognitivos, metacognitivos, motivacionales y conductuales.

El estudio de Cerezo, Bernardo, Esteban, Sánchez y Tuero (2015) concluye que los programas destinados a fomentar la autorregulación del aprendizaje en modalidad virtual sobre la presencial implican una ganancia mayor en las variables, interés, claridad y aplicabilidad del material, utilidad y reflexión en la forma de estudiar. Diferencias observadas que derivan en una mayor motivación, implicación o participación activa del estudiante en contextos virtuales.

En suma, se supone que tanto la modalidad educativa presencial como virtual muestran particularidades sobre el clima de la clase y el compromiso académico percibido por los estudiantes. Se entiende que, los resultados permitirán trazar una definición que contribuya a concretar contextos educativos propicios para estudiantes más comprometidos postpandemia.

\section{Metodología}

En el estudio desarrollado de tipo descriptivo, bajo lineamientos metodológicos mixtos, se propuso describir cómo los estudiantes se comprometen, en contextos académicos presenciales y virtuales, y a la vez cómo perciben el clima de la clase en cada entorno. Asimismo, se buscó indagar sobre las actividades de aprendizaje más significativas, ya sea por su modalidad, contenido o contexto en la cual se desarrolló, junto a las dimensiones del clima de la clase que contribuyen o no a sus intereses, participación, autorregulación y expresión de perspectivas. 


\subsection{Participantes}

El primer estudio se desarrolló en el 2019, durante el segundo cuatrimestre. Participaron estudiantes de la Universidad Nacional de Río Cuarto, Córdoba, Argentina, de dos Licenciaturas, Educación Inicial ( $n=56$ ) y Educación Física $(n=46)$, contando un número total de 102 estudiantes, con predominio de mujeres ( $71 \%$ ). La edad media fue de 20,55 años $(S D=3,7)$, lo que caracteriza a un grupo que se encontraba cursando materias de los primeros años de sus respectivas carreras, Creatividad del primer año de plan de estudio del Profesorado en Educación Inicial y Didáctica del segundo año del Profesorado en Educación Física.

El segundo estudio se llevó a cabo en el marco del primer cuatrimestre del año 2020, dictado en su totalidad bajo la modalidad virtual. Participaron 109 estudiantes matriculados en tres instituciones de nivel superior de la ciudad de Río Cuarto, Argentina, a saber: Universidad Nacional de Río Cuarto, Universidad de Mendoza e Instituto Superior María Inmaculada. Los participantes se encontraban estudiando las siguientes carreras: Licenciatura en Psicopedagogía $(n=28)$, Licenciatura en Comunicación Social $(n=2)$, Licenciatura en Educación Inicial $(n=25)$, Licenciatura o Profesorado en Psicología ( $n=22)$, Profesorado en Ciencias Políticas y Jurídicas, Abogacía $(n=17)$ y Radiología e Instrumentador Quirúrgico $(n=15)$. Las edades estuvieron comprendidas entre los 17 y los 35 años, con un promedio de 22,3 años $(S D=4,1)$, siendo casi la totalidad mujeres, a excepción de tres varones.

La selección en ambos estudios se realizó bajo un muestreo casual (Padua, 1979), en tanto se seleccionó directa e intencionadamente a los estudiantes que estuvieran cursando alguna materia con facilidad de acceso. Se informó a los participantes sobre los objetivos y los alcances del estudio aprobado por el comité de ética de la investigación (COEDI) de la Universidad Nacional de Río Cuarto, Argentina. También se les comunicó respecto a la confidencialidad de los datos y la preservación del anonimato. Los estudiantes dieron consentimiento informado para la realización del estudio y la publicación de resultados.

\subsection{Instrumentos}

Para la recolección de datos, se utilizaron tres instrumentos, dos de los cuales fueron adaptados o elaborados por el equipo de investigación a los fines de atender a los criterios de validez y confiabilidad para nuestra población y contexto de estudio.

El primero se utilizó para valorar la percepción de los estudiantes acerca de su compromiso académico, es un cuestionario que integra las cuatro dimensiones del constructo: afectiva, cognitiva, conductual y de agencia personal. Para indagar sobre las tres primeras, se tomaron los ítems del University Student Engagement Inventory (USEI) desarrollado por Maroco, Maroco, Campos y Fredricks (2016), mientras que, para la última, se retomaron los aportes de Veiga (2013). En total, el cuestionario tiene 20 ítems, cinco por dimensión, todos precedidos por la frase 'durante la clase', cuyas opciones de respuestas en formato Likert de 6 puntos marcan el acuerdo o el desacuerdo con cada proposición. Con relación a la validez y confiabilidad, se encontraron resultados aceptables para una estructura de cuatro dimensiones $\left(X^{2}=283,02\right.$, $\left.g l=164, p=, 0001, X^{2} / g l=1,72, \mathrm{CFI}=, 80, \mathrm{GFI}=, 81, \mathrm{RMSE}=, 083-\mathrm{IC} 90 \%=, 066 /, 098-, \alpha=, 74\right)$, mediante análisis factorial confirmatorio usando AMOS v. 23 y coeficiente alpha de Cronbach. Cabe aclarar que los ítems no fueron modificados, se realizó una breve introducción en el estudio 2020, explicando la importancia de atender a las circunstancias actuales de cursado.

El segundo denominado Evaluar el Clima de Aula Universitaria, diseñado y validado por Ríos et al. (2010), es un cuestionario que solicita a los estudiantes que lean una oración y puedan establecer el grado de acuerdo o desacuerdo -escala Likert de 6 puntos- de 41 afirmaciones con respecto a 7 dimensiones presentes en el clima de aula: infraestructura ( 2 ítems), equipamiento (5 ítems), dominio de contenido (5 ítems), metodología de la clase (9 ítems), práctica evaluativa 
(9 ítems), la relación profesor-estudiante ( 6 ítems) y la relación entre estudiantes ( 5 ítems). Un análisis factorial confirmatorio, realizado con AMOS v. 23, reveló resultados óptimos para una estructura bifactorial $\left(X^{2}=17,47, p=, 1330, \mathrm{CFI}=, 94, \mathrm{GFI}=, 98, \mathrm{RMSE}=, 077-\mathrm{IC} 90 \%=, 01 /, 15-\right)$ y la consistencia interna arrojó un valor adecuado de $\alpha=, 82$. Durante su administración en el presente año, se explicitó en un breve párrafo que "infraestructura" hacía alusión a los espacios disponibles en el hogar, y que "equipamiento" hacía a los dispositivos tecnológicos disponibles para acceder a una clase virtual, así como conexiones a internet.

Por último, se les solicitó a los estudiantes por escrito, mediante un cuestionario ad hoc de preguntas abiertas, que indicarán algunas de las actividades de aprendizaje propuestas por los docentes a cargo de la asignatura que fueron interesantes, ya sea por su modalidad, contenido o contexto en la cual se desarrolló. Fundamentando la elección y mencionando aspectos del clima de la clase que contribuyeron o no a sostener sus intereses, participar, autorregular el propio proceso de aprendizaje y expresar sus perspectivas.

\subsection{Procedimiento}

La entrada a las aulas de las diferentes asignaturas se realizó solicitando autorización a los profesores responsables de las asignaturas. La recolección de datos durante el 2019, se llevó a cabo los días pautados y acordados para no interferir en el dictado de las clases. Mientras que, durante el 2020, los cuestionarios fueron enviados a través de Google Formulario ${ }^{\circledR}$. En cada instancia, se solicitó al estudiante que pensara en la asignatura en la cual se estaban administrando los instrumentos y respondiese a continuación. El tiempo que demandó la tarea se promedia en una hora.

La administración, en ambos casos, se efectuó cerca del final de cada materia, para que pudieran tener los estudiantes una visión general de cada elemento que define el clima de aula. Los datos recolectados a partir de los instrumentos estandarizados se cargaron en una matriz de datos y cada protocolo se codificó para resguardar el anonimato de los estudiantes. Mientras que, los datos provenientes del cuestionario ad hoc se guardaron en documentos de tipo texto.

\subsection{Análisis de datos}

Para el análisis de datos, se utilizó el programa estadístico SPSS versión 19 para llevar a cabo el análisis cuantitativo de datos usando estadísticos de tendencia central (Sampieri, Collado y Lucio, 2010). Asimismo, se efectuó la prueba Kolmogorov-Smirnov y se constató la presencia de una distribución no normal para cada una de las dimensiones de clima del aula y compromiso ( $p$ $<.05)$, por lo que se aplicó la Prueba U de Mann-Whitney para extrapolar los resultados a toda la población. La significación estadística se estableció con un valor de $p \leq .05$. Para el análisis cualitativo se usó el ATLAS.ti 8, específicamente, las nubes de palabras, una herramienta que sintetiza la relación frecuencia de aparición con el tamaño de la palabra y constituye un recurso que permite iniciar el proceso de codificación y la elaboración de categorías (Hernández Fernández, 2015).

\section{Resultados}

Iniciando por la variable clima de la clase, los Gráficos 1 y 2 sintetizan las medianas para cada una de las 7 dimensiones valoradas durante el año 2019 y 2020, respectivamente, a saber: infraestructura, equipamiento, contenido, metodología, evaluación, relación profesoresestudiantes (RPE) y relación estudiantes-estudiantes (REE). En el Gráfico 1 se observa la puntuación igual o mayor que el $50 \%$ de los estudiantes obtuvieron cuando informaron sus percepciones sobre la configuración del clima de la clase en aulas físicas. 
Gráfico 1

Mediana para cada dimensión del clima de la clase, durante el año 2019

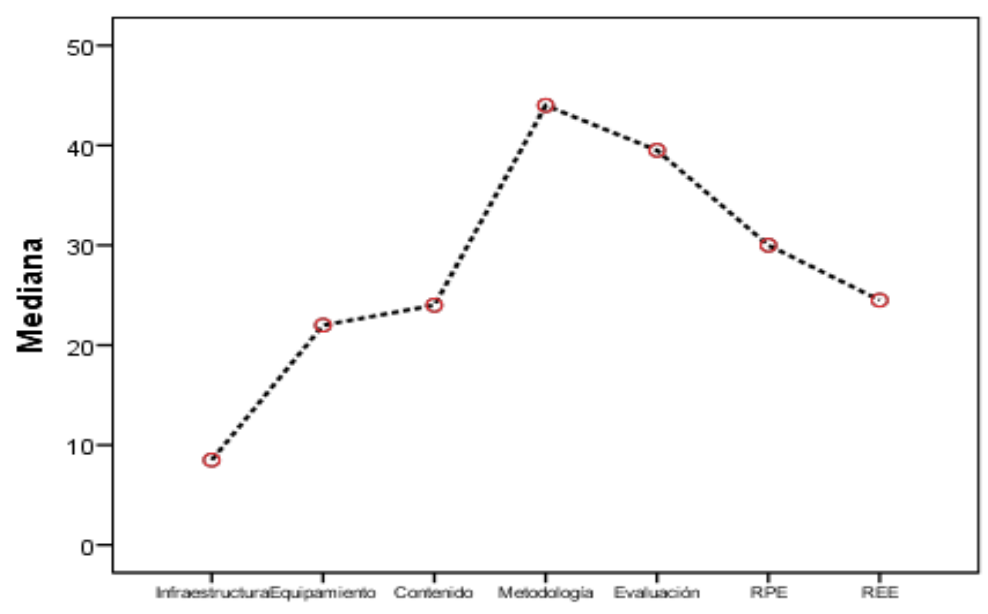

Fuente: Elaboración propia

Sin embargo, en el Gráfico 2 se presenta la misma información de los estudiantes que cursaron en modalidad virtual, observándose que, en todas las dimensiones, menos en equipamientos, las puntuaciones son mayores si se las compara con las alcanzadas por los estudiantes en el año 2019. Encontrándose diferencias estadísticamente significativas para todas las dimensiones de clima de la clase entre los estudiantes que cursaron presencial y los que lo hicieron de manera virtual, a excepción de la dimensión equipamiento, como se puede observar en la Tabla 1.

Grafico 2

Mediana para cada dimensión del clima de la clase durante el año 2020

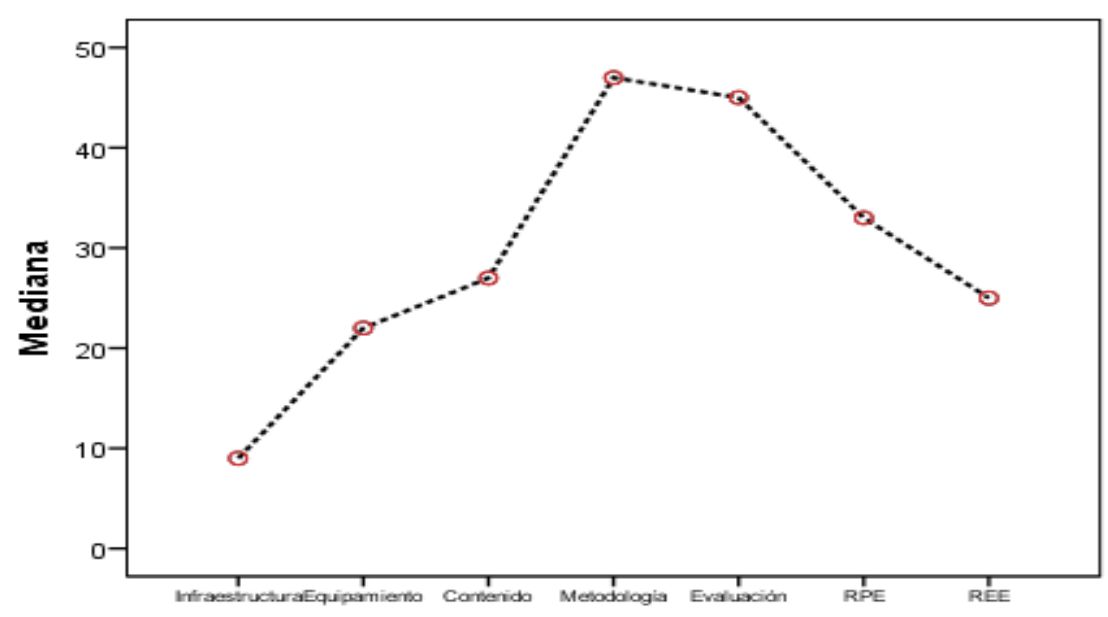

Fuente: Elaboración propia 
Tabla 1

Prueba U de Mann-Whitney para las dimensiones del clima del aula entre el año 2019 y el año 2020

\begin{tabular}{lccc}
\hline \multicolumn{1}{c}{ Dimensión } & Año & Rango Promedio & U (Sig.) \\
\hline Infraestructura & 2019 & 82.45 & $\begin{array}{c}3156.5 \\
(.023)\end{array}$ \\
\cline { 2 - 3 } & 2020 & 100.01 & $\begin{array}{c}4135.5 \\
(.537)\end{array}$ \\
\hline Equipamiento & 2019 & 92.07 & 2324.5 \\
& 2020 & 87.25 & $(.000)$ \\
\hline Contenido & 2019 & 74.29 & 2705.0 \\
& 2020 & 110.81 & $(.000)$ \\
\hline Metodología & 2019 & 78.02 & 2317.0 \\
& 2020 & 105.87 & $(.000)$ \\
\hline Evaluación & 2019 & 74.22 & 2625.0 \\
& 2020 & 110.91 & $(.000)$ \\
\hline RPE & 2019 & 77.24 & 3155.5 \\
& 2020 & 106.91 & $(.025)$ \\
\hline REE & 2019 & 82.44 & \\
\cline { 2 - 3 } & 2020 & 100.02 & \\
\hline
\end{tabular}

Los aspectos positivos y negativos del clima de la clase que mencionaron los estudiantes que participaron del estudio permitieron comprender en mayor profundidad el significado de los valores graficados. En la Figura 1, se agrupan en dos nubes dichos aspectos mencionados por los estudiantes durante el 2019.

Figura 1

Palabras más repetidas en las respuestas de los estudiantes sobre el clima de la clase presencial
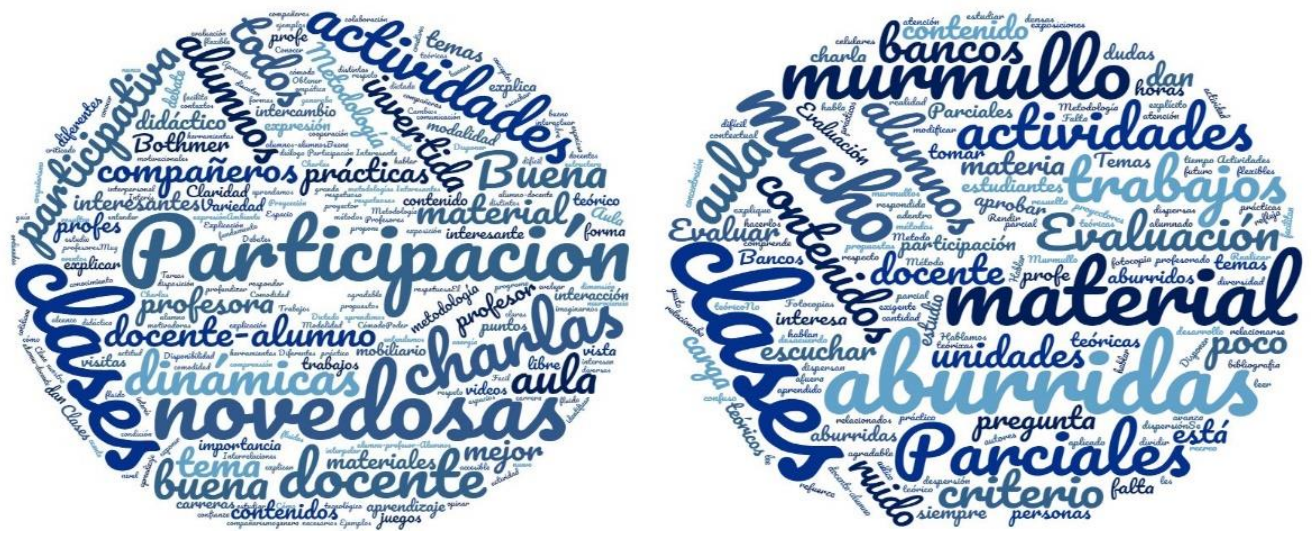

Fuente: Elaboración propia

Como se aprecia, durante el 2019 los estudiantes rescataron diversos aspectos del clima de la clase que propiciaron sus intereses, participación, autorregulación y expresión de perspectivas. La nube izquierda muestra los positivos, y entre ellos, se destacaron actividades novedosas o que salieron de la rutina, como la incorporación de las TIC -Tecnologías de la Información y la Comunicación- y contextos extra clase como las charlas con profesionales, la relación docente y estudiantes como medio para aprender, incluso el compañerismo como elemento central de la clase que dinamiza de participación y debate. Entre los aspectos negativos, sobre la nube ubicada del lado derecho, se aprecia que los estudiantes remarcaron el murmullo en las clases, las evaluaciones caracterizadas como muy teóricas, con poco vínculo con el futuro rol 
profesional, la carga de material valorado y la ausencia de criterios claros de valoración, como así también los contenidos impartidos en las clases, asimilando la presencia de material aburrido para leer o bien presentado por la docente de manera monótona.

Debajo, en la Figura 2, aparecen las palabras más utilizadas por los estudiantes para expresar el clima de la clase virtual. Donde se aprecia que, las evaluaciones como proceso formativo, divergente, múltiple y flexible se percibieron positivamente. Asimismo, las clases grabadas como posibilidad de acceder en tiempos distintos a los pautados, surfeando dificultades de conectividad, coordinando maternidad y compromisos laborales se remarcaron entre las palabras mencionadas con mayor frecuencia. Además, las retroalimentaciones en los grupos de WhatsApp entre compañeros como medio para seguir conectados y lograr apoyos mutuos en la virtualidad y aprender a ser estudiantes remotos, fueron conceptos que resumieron los aspectos positivos del clima que aparecieron sobre la nube izquierda. No obstante, sobre la nube derecha, los estudiantes mencionaron aspectos negativos, la presencia de muchas actividades simultáneas de distintas materias, en ausencia o falta de coordinación inter-cátedras, el coordinar los tiempos y espacios del hogar, la dificultad de acceder a clases sincrónicas por conexiones a internet inestables o débiles, sumando la complejidad de interaccionar con los docentes y pares de manera fluida, junto a los temores por participar e interrumpir el desarrollo de la clase, de no saber cómo y cuándo hacerlo en educación remota.

Figura 2

Palabras más repetidas en las respuestas de los estudiantes sobre el clima de la clase virtual
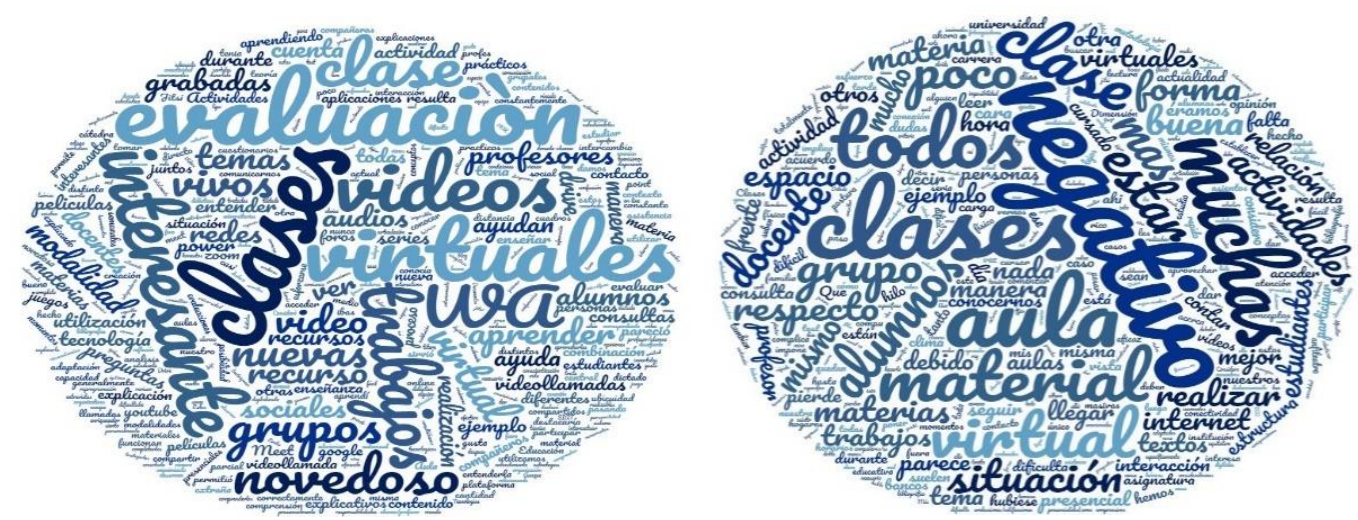

Fuente: Elaboración propia

En relación con la variable compromiso académico percibido por el alumnado, los análisis arrojaron una puntuación promedio de $17,40(S D=5,38)$ para la dimensión agencia, de 23,14 $(S D=3,37)$ para la conductual, de $19,76(S D=4,13)$ para la afectiva y, por último, de 23,30 $(S D=4,44)$ para la cognitiva, esto para el año 2019. Durante el 2020, los estudiantes en promedio se percibieron comprometidos un $20,72(S D=4,27)$ en la dimensión agencia, un $25,94(S D=2,82)$ en lo conductual, un $22,84(S D=2,29)$ en lo afectivo y un $25,67(S D=2,83)$ a nivel cognitivo. Para cada dimensión, se encontraron diferencias estadísticamente significativas, como se aprecia en la Tabla 2. 
Tabla 2

Prueba U de Mann-Whitney para las dimensiones de compromiso académico entre el año 2019 y el año 2020

\begin{tabular}{lccc}
\hline Dimensión & Año & Rango Promedio & U (Sig.) \\
\hline \multirow{2}{*}{ Agéntico } & 2019 & 87.0 & 7497.0 \\
& 2020 & 123.78 & $(.000)$ \\
\hline Conductual & 2019 & 78.10 & 8404.5 \\
& 2020 & 132.11 & $(.000)$ \\
\hline Afectivo & 2019 & 81.39 & 8069.0 \\
& 2020 & 129.03 & $(.000)$ \\
\hline Cognitivo & 2019 & 87.98 & 7397.5 \\
& 2020 & 122.87 & $(.000)$ \\
\hline
\end{tabular}

Entre las expresiones y voces de los estudiantes se mencionaron diversas actividades de aprendizaje que se señalaron como más significativas, contemplando su modalidad, contenido y contexto de realización. En las siguientes nubes de palabras se sinterizaron (Figuras 3 y 4), las palabras más mencionadas tanto en el año 2019 como en el 2020.

Figura 3

Palabras más repetidas en las respuestas de los estudiantes (2019) sobre las actividades que más comprometieron

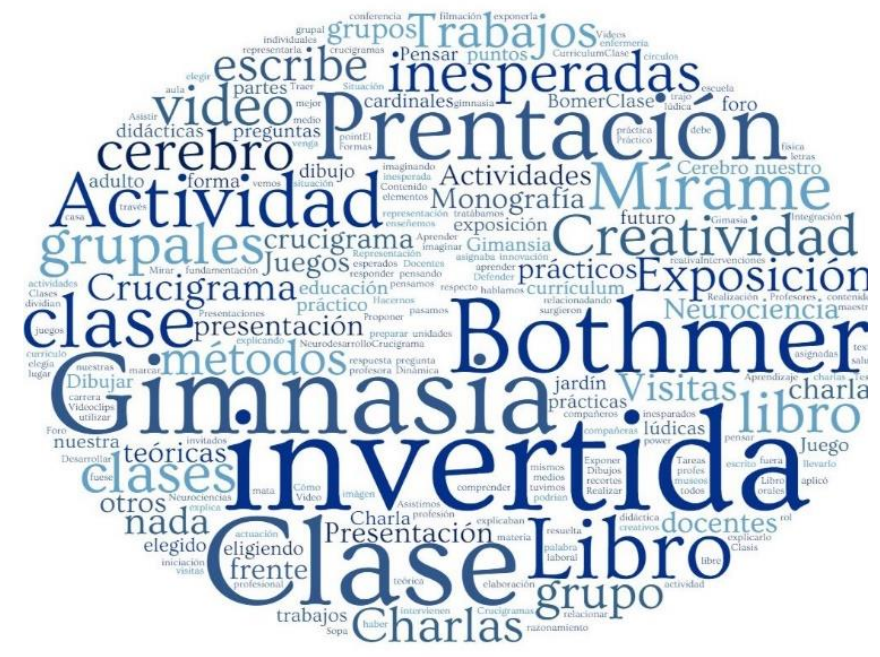

Fuente: Elaboración propia 
Figura 4

Palabras más repetidas en las respuestas de los estudiantes (2020) sobre las actividades que más comprometieron

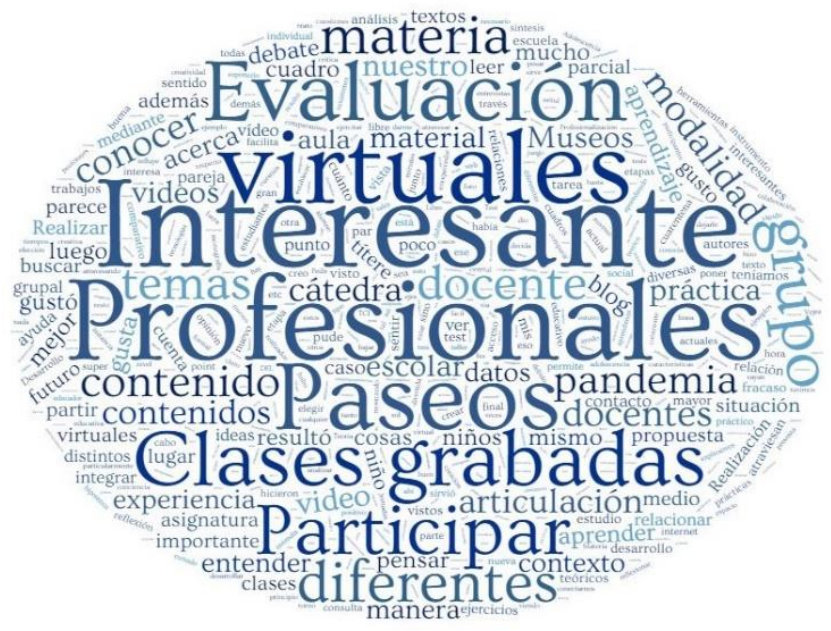

Tanto en el 2019 como en el 2020, los estudiantes mostraron afinidad por tareas académicas que parecen captar un mayor compromiso con sus aprendizajes y que guardan vínculo con los aspectos positivos del clima de la clase. Esas actividades tienen dos aspectos que aparecen como claves en la literatura para promover el compromiso, educación expandida y metodologías activas (Rigo y Guarido, 2020). Por ejemplo, mencionaron el enfoque de clase invertida y charlas con otros profesionales en otro contexto distinto al aula, tales como, un curso de gimnasia Bothmer en el gimnasio mayor de la universidad o la presentación de un libro en la biblioteca central del campus universitario. Debates y presentaciones que no sólo se resaltaron durante el 2019 , sino que aparecieron bajo nuevas modalidades durante el 2020, tales como, conferencias en vivo, explicaciones grabadas del docente disponibles en aulas virtuales y paseos virtuales. Nuevamente, aparecieron las interacciones entre docentes y pares en ambos contextos de estudio y, la evaluación formativa y el grupo de compañeros, como aspectos vinculados con las percepciones del compromiso asumido en una educación remota.

Entre los fundamentos de los aspectos positivos tanto del clima de la clase como de aquellos que promovieron el compromiso percibido, los estudiantes mencionaron indicadores clave de una agencia expandida, tal cual lo plantean Rowlands (1997), Rojas Carvajal (2015) y Samman y Santos (2009).

En primera instancia aparecieron menciones que aluden a un poder junto a otros que ayuda a moldear el contexto instructivo y los eventos que suceden en torno a las tareas académicas para resolver de manera conjunta alguna problemática. La capacidad de cambiar y hallar soluciones junto a otros en la comunidad educativa de pertenencia se reflejó en las siguientes expresiones:

- "Pedir la colaboración de colegas mediante WhatsApp y nada más que por ahí, sin ningún tipo de contacto fue algo nuevo" (105-2020).

- "Considero como novedoso la utilización de la red social 'WhatsApp' para sostener el vínculo docente-alumno durante la pandemia" (76-2020).

- "Me sirvió mucho participar de foros donde se debatieron distintos temas y también la creación de un grupo de WhatsApp con todas las personas que hacíamos la clase" (982020).

- "Probar infinidad de plataformas hasta encontrar una en la que todos podrían acceder, escuchar y atender la clase, respetando las diferencias" (25-2020). 
- "Las tecnologías, es un recurso que yo no tenía muy en cuenta, y realmente nos permiten estar en contacto con nuestros compañeros y profesores para realizar un aprendizaje dinámico y en esta pandemia solucionar el dictado de clase" (12-2020).

- "...resulta interesante que graben las clases lo cual me parece muy positivo ya que en muchos casos dadas las circunstancias de cada uno no podemos acceder entonces de esa forma no perdemos la clase (58-2020).

- "La clase invertida nos permitió conocer distintas alternativas de trabajo. Fomentar la cooperación, libertad y solidaridad entre compañeros, encontrar soluciones a problemas que vamos a tener que enfrentar como futuros profesionales en las escuelas y la sociedad" (56-2019).

- "Ver videos, tener charlas con profesionales nos permite descubrir nuevas formas de educación. Descubrir diferentes realidades en educación, pensar la teoría en la práctica, al igual que las prácticas socio-comunitarias donde de manera conjunta buscamos soluciones con la comunidad" (14-2019).

- "Reflexión acerca de la profesionalización docente. En este caso fue muy positivo porque pensar en la práctica docente y la importancia de su carácter profesional, la necesidad de estar en constante formación cobró mayor sentido en este contexto de aislamiento social y obligatorio" (66-2020).

En segunda instancia emergieron comentarios que referían a un poder para crear nuevas oportunidades, actuando con autonomía y tomando decisiones frente al grupo de pertenencia. Es decir, poder expresar la opinión propia, elegir y participar en las opciones dispuestas en la comunidad educativa de pertenencia. Las voces de los estudiantes así lo manifestaron:

- "Uso del celular... descargamos una app, escaneamos un documento presentado por el docente y realizamos una reflexión grupal sobre la temática que debíamos elegir. Me pareció una forma diferente de encarar la clase, y además me sentí cómoda trabajando grupalmente, en donde el grupo era de a dos alumnos; considero que así pudimos trabajar más amenamente y mejor. Y participar desde nuestras opiniones" (74-2020).

- "Es un desafío, las clases virtuales, ya que implican más autonomía a la hora de desarrollar y comprender contenido" (99-2020).

- "El debate entre compañeros y docentes que aporta al entendimiento sobre un determinado tema, y a su vez, permite conocer diferentes posturas, expresar la nuestra" (55-2020).

- “...no siempre ser evaluado con una nota, sino de forma crítica con devoluciones contractivas, porque muchos tienen miedo a participar por equivocarse. Abrir espacios para escuchar, plantear y proponer en la clase nos permite crear un ambiente más ricos de aprendizaje" (86-2020).

- "Los intercambios en Facebook, durante la clase invertida, nos permitieron a todos los integrantes de cada grupo expresar su opinión, más interacción y diversidad de ideas" (95-2019).

- "Entender que podemos ser autónomos, elegir temática a investigar, buscar información sin esperar a que la dé el profesor, eso nos da libertad de elección y toma de decisiones en nuestro proceso de aprendizaje" (106-2019).

- "Reflexiones sobre cómo nos sentimos ante esta situación de pandemia" (56-2020).

En tercera instancia un poder sobre los recursos vinculados a la capacidad de poder controlar las decisiones propias del día a día, vinculadas al concepto de autoeficacia. Los estudiantes así lo manifestaron cuando percibieron que pudieron finalizar las actividades, gestionar y organizar el tiempo con responsabilidad: 
- "En cuanto a lo material, es muy positivo aprender en lo que podemos construir grandes cosas con las pequeñas cosas que podemos encontrar en casa, construir dentro de un plano una imagen hermosa, lleno de materiales allá adentro que construyen una perspectiva diferente, dependiendo del punto de vista de cada uno" (29-2020).

- "Es muy diferente, se extraña mucho el contacto que hay en el aula, los vínculos que se crean, la interacción que esta permite. Igualmente, la posibilidad de tener clases virtuales, que los docentes nos manden el material y la predisposición de ellos para que no perdamos el cuatrimestre, creo que también nos incentiva a dar lo mejor de nosotros mismos, a proponernos avanzar en el estudio, aunque no se tenga la presión de una fecha examen, a proponerse cumplir con todas las tareas a tiempo y prestar atención a cada clase, por más que estemos en nuestras casas, pensar que estamos en el aula y dar lo mejor" (70-2020).

- "Aprender en cuarentena demanda de mucho tiempo, al principio es difícil ya que no estaba acostumbrada a la modalidad virtual y se complica acordar horarios de estudio con el resto de la familia por lo que algunas veces se tornaba molesto estar en clases y que el resto de los integrantes del hogar tengan que adaptarse de un día para el otro a nuestros horarios de estudio en casa pero luego que pasa el tiempo, he podido organizar mis momentos de estudio y he podido participar mucho más en las clases debido a que ya había confianza entre profesores y compañeras" (103-2020).

- "El recurso que me pareció interesante fue la de grabar la clase me parece que permite al estudiante volver en aquello que no se comprendió en los tiempos del hogar, la familia y los hijos" (104-2020).

- "Trabajar con modelos mixto de enseñanza y aprendizaje me facilito organizarme, poder acceder al material, a los videos desde casa, en el tiempo que podía y organizar las actividades sin necesidad de esperar la clase de consultar presencial, entraba a Facebook y podía interactuar para avanzar en el momento que tenía para hacerlo" (1592019).

- "Son metodologías que nos motivan a terminar la carrera, nos hacen sentir más autónomos, qué podemos hacer las cosas por sí mismo, sin la presencia del profesor constante, que tenemos habilidades para desempeñarnos, pensar y planificar una clase" (148-2019).

- "Es aprender de manera diferente, en momentos diferentes, donde hoy a partir de la situación que estamos atravesando nos consideramos y los docentes se consideran sujetos de aprendizaje". Esta frase la tomé de una charla que tuve ayer donde se remarcaba todo el tiempo que el aprendizaje está a pesar de la distancia, donde si hubo un cambio de escenario, de contexto, pero que el aprendizaje en cuarentena sigue estando. Que es un gran desafío para todos, lo es porque es el momento en donde ponemos a prueba nuestras capacidades y a la vez fortalecemos nuestras propias creencias que se nos presenta en dicha situación" (96-2020).

Por último, un poder interno, como habilidad para cambiar a nivel personal. Capacidad que los estudiantes pusieron en palabras cuando se refirieron al momento de adaptarse a la vida universitaria, aprender a ser estudiantes remotos y tomar decisiones en vista a cumplir metas de largo plazo:

- "Considero que es una gran y nueva experiencia incierta y enriquecedora aprender en este contexto, pero también lo veo como una gran oportunidad de cambiar formas de enseñanza, aprendizaje y de ver y reflexionar acerca de determinadas situaciones 0 conflictos que surgen" (83-2020).

- "Creo que es todo un desafío donde muchos son los factores que se ponen en juego; sin embargo, creo que el punto en común es que hay esfuerzos provenientes de todos 
lados. Me gustaría resaltar un aprendizaje muy valioso desde lo personal, que es la importancia de entender que más allá de mi situación particular, existen otras totalmente diferentes que también deben ser contempladas, atendidas y escuchadas; así como los esfuerzos de cada persona en lo individual, familiar, social, educativo, psicológico, etc." (95-2020).

- "Es un desafío, implica nuevas modalidades, modos de relación, herramientas de estudio, etc. A su vez genera nuevos aprendizajes. Nos desafía a ser distintos como estudiantes, ser más responsables de nuestros tiempos y espacios (106-2020).

- "Creo que el mayor aprendizaje en estas clases fue definir cómo quiero ser profesora el día de mañana, cambiar esa idea que solo podemos enseñar con la tiza y el pizarrón" (123-2019).

- “...con los diversos contactos con profesionales, charlas y demás comprendí el valor de la carrera que estoy cursando, cambie mi perceptiva acerca de lo que voy a ser a futuro, creo que no sabía muy bien cuál era mi objetivo. Empecé a estudiar para cumplir con una pauta familiar, hoy es mi objetivo" (100-2020).

\section{Discusión y conclusiones}

El objetivo general de esta investigación era describir cómo los estudiantes se comprometen, en contextos académicos presenciales y virtuales, y a la vez cómo perciben el clima de la clase en cada entorno. Asimismo, se buscó indagar sobre las actividades de aprendizaje y las dimensiones del clima de la clase que más contribuyen a sus intereses, participación, autorregulación y expresión de perspectivas.

Los resultados cuantitativos obtenidos muestran que las dimensiones del clima del aula y del compromiso académico se perciben significativamente distintas cuando los estudiantes cursan de manera presencial o virtual. Específicamente, son los estudiantes que cursaron en pandemia los que tienen una percepción más ajustada de las dimensiones inmateriales y materiales del clima de la clase, con excepción de los equipamientos disponibles para el desarrollo de la clase. Asimismo, son estos mismos estudiantes quienes obtuvieron puntuaciones medias superiores en las diversas manifestaciones del compromiso académico valoradas con respecto al grupo que cursó en el 2019 presencialmente. La investigación de Macchiarola, Pizzolitto, Pugliese y Muñoz (2020) muestra que la falta de acceso a dispositivos tecnológicos, las dificultades para acceder a dispositivos compartidos familiarmente y la mala conectividad son las principales problemáticas identificadas por los estudiantes en pandemia.

Con respecto a los análisis cualitativos, los estudiantes manifestaron que ciertas actividades son más afines a promover el compromiso y establecer un mejor clima de la clase. Concretamente, las clases que se expanden hacia otros contextos y enfoques fueron valoradas como positivas. En específico mencionaron la flipped classroom y el salir de la clase como nuevos escenarios educativos que aportan un diálogo entre la teoría y la práctica. Uribe-Zapata (2018) al respecto plantea que el aprendizaje en la educación expandida puede darse en cualquier momento y situación, implicando un trabajo colaborativo y de intercambio de información abierta y flexible, donde el estudiante se piensa como agente activo y responsable de su propio proceso de aprendizaje. Asimismo, el estudio de Schindler, Burkholder, Morad y March (2017) encuentra que los contextos expandidos se correlacionan positivamente con el compromiso estudiantil. No obstante, también resalta que los problemas asociados a la falta de una adecuada infraestructura o requisitos tecnológicos pueden socavar la participación y la motivación de los estudiantes. Aspectos, éstos últimos, que aparecieron valorados negativamente dentro del clima de la clase entre los estudiantes que participaron del estudio encaminado durante el 2020. 
Son las evaluaciones el aspecto que aparece como antagónico y el gran reto a repensar del clima de la clase. Durante la educación virtual 2020 se pondera la necesidad de repensar formatos más tradicionales, que desde la presencialidad aparecían como menos valorados por los estudiantes. Cappelletti (2020) propone que la enseñanza, el aprendizaje y la evaluación se tratan de un recorrido, no de procesos separados. La evaluación formativa supone narrar el recorrido realizado por los estudiantes a través de sus producciones, analizar logros, generar sugerencias, formular caminos alternativos para alcanzar lo que aún no se pudo. Favorecer este tipo de valoraciones es andamiar la autorregulación y la participación de los estudiantes en sus procesos de aprendizaje.

Además, los resultados muestran que atender a metodologías más activas de enseñanza y aprendizajes es fundamental para que los estudiantes logren no solo interesarse, participar más y gestionar sus aprendizajes, sino también exteriorizar sus agencias. Sobre esta última dimensión del compromiso, la agéntica, los resultados en la perceptiva de los estudiantes muestran que un clima de aula que favorece la autonomía, la responsabilidad, la toma de decisiones, la búsqueda de metas personales y la participación, es coincidente con la posibilidad de interactuar con el contexto que los rodea y hacer que las cosas suceden en pos de estar más implicados con sus aprendizajes académicos. Lo cual coincide con lo formulado por Narayan (2005), Samman y Santos (2009), Pick et al. (2007) y Rowland (1997).

Los aportes del presente estudio también muestran la importancia de los vínculos entre docentes y estudiantes. Resultado que concuerdan con la investigación de Macchiarola et al. (2020) y Morán (2020) quienes señalan la importancia de considerar y revisar las estrategias de enseñanza, promoviendo pedagogías más interactivas y menos transmisoras para promover la comunicación, como el uso de WhatsApp, para fortalecer el diálogo entre los profesores con los estudiantes y entre ellos. Asimismo, los estudiantes valoraron positivamente las instancias de participación activa que apoyaron sus procesos de autorregulación y de agencia, tanto en contextos presenciales como virtuales. Aspecto que coincide con las conclusiones de Manzuoli, Pineda-Baez y Sanchez (2019).

Los resultados encontrados, tanto en el estudio desarrollado en el 2019 como el realizado en el 2020, muestran algunas tendencias que permiten delimitar aspectos claves a tener presente en la definición de una nueva pedagogía con clara intención de generar un clima de la clase tendiente a promover el compromiso de los estudiantes en el nivel superior de educación postpandemia. Contemplando el antes y el durante de una educación en contextos de emergencia, e impactar en los procesos de desvinculación, que tal como estima Claus (2020) en Argentina rondará en 1,5 millones de estudiantes.

En próximos estudios, los análisis deben atender a comprender en mayor profundidad la tendencia que aparece en los datos presentados, contemplando un mayor tamaño muestral representativo de la población objeto de estudio. Sería pertinente seguir indagando las percepciones de los estudiantes sobre el compromiso percibido y el clima de la clase durante el próximo año académico para seguir marcando una futura agenda educativa. Asimismo, atender a una definición más contextualizada de los instrumentos aplicados en clases virtuales tanto para valorar el clima de la clase como el compromiso percibido por los estudiantes.

En síntesis, la pandemia con su novedad, y la casi obligada tarea de salir de la rutina ha sido un aliciente para seguir renovando las prácticas educativas en educación superior. Salir de la clase, buscar innovar junto a las TIC, tener mayores vínculos con las comunidades profesionales y sociales de cara al desempeño profesional, son los nuevos territorios a recorrer en una educación superior que se repiensa en transición. 


\section{Referencias}

Arguedas Negrini, I. A. (2010). Involucramiento de los estudiantes y las estudiantes en el proceso educativo. Revista Iberoamericana sobre Calidad, Eficacia y Cambio en Educación, 8(16), 3-78.

Cappelletti, G. (2020). Cuatro afirmaciones sobre evaluar para aprender que deberíamos contemplar en tiempos de pandemia. Panorama OEl. https://panorama.oei.org.ar/cuatro-afirmaciones-sobre-evaluar-para-aprender-entiempos-de-pandemia/

Cerezo, R., Bernardo, A., Esteban, M., Sánchez, M. y Tuero, E. (2015). Programas para la promoción de la autorregulación en educación superior: un estudio de la satisfacción diferencial entre metodología presencial y virtual. Revista Europea de Educación y Psicología, 8(1), 30-36. https://doi.org/10.1016/j.ejeps.2015.10.004

Chiecher, A y Donolo, D. (2006). Búsqueda de ayuda en ambientes presenciales y virtuales. XIII Jornadas de Investigación y Segundo Encuentro de Investigadores en Psicología del Mercosur. Universidad de Buenos Aires.

Cimadevilla, G. (2020). Interrogantes en tiempos de educación virtual. Question/Cuestión, 1 (mayo), 1-5. https://doi.org/10.24215/16696581e281

Claus, A. (2020). ¿Cuántos estudiantes dejarían la escuela en la Argentina a causa del coronavirus? https://chequeado.com/hilando-fino/cuantos-estudiantes-dejarian-laescuela-en-la-argentina-a-causa-del-coronavirus/

Deng, R., Benckendorff, P. y Gannaway, D. (2020). Learner engagement in MOOCs: Scale development and validation. British Journal of Educational Technology, 51, 245-262. https://doi.org/10.1111/bjet.12810

Dreesman, H. (1982). Classroom climate: contributions from a European country. Studies in Educational Evaluation, 8, 53-64.

Fanelli, A., Marquina, M. y Rabossi, M. (2020). Acción y reacción en época de pandemia: la universidad argentina ante la COVID-19. ESAL. Revista de Educación Superior en América Latina, http://rcientificas.uninorte.edu.co/index.php/esal/article/viewFile/13401/21442144489

Fernández-Ballesteros, R. y Sierra, B. (1989). Estudios factorial sobre la percepción del ambiente escolar. En R. Fernández-Ballesteros (Coord.). Intervención psicológica en contextos ambientales (pp. 143-176). Servicio de Publicaciones de la Universidad de Murcia.

Fredricks, J. A., Blumenfeld, P. C. y Paris, A. H. (2004). School engagement: Potential of the concept, state of the evidence. Review of Educational Research, 74, 59-109.

Galini, R. y Efthymia, P. (2009). Dimensions of the classroom climate, as perceived by the students, related to their teachers' evaluation approach on their overall performance in a Greek primary school sample. New Horizons in Education, 57(2), 109-120.

García de Fanelli, A. (2007). Acceso, abandono y graduación en la educación superior argentina. Sistema de información de tendencias educativas en América Latina, 5, 1-17. http://www.vcn.siteal.org/sites/default/files/siteal_debate_5_fanelli_artculo.pdf

González González, M. (2010). El alumno ante la escuela y su propio aprendizaje: algunas líneas de investigación en torno al concepto de implicación. Revista Iberoamericana sobre Calidad, Eficacia y Cambio en Educación, 4, 10-31. 
Harrison, T. (2014). Conceptualizing student engagement: A co-creation perspective. En D. Derricott (Edit.). Working together to take quality forward. A selection of papers from the 8th European Quality Assurance Forum (pp.51-58). European University Association.

Heedy, C. y Uribe, M. (2008). La educación a distancia: sus características y necesidad en la educación actual. Educación, XVII(33), 7-27.

Hernández Fernández, C. (2015). Nuevos recursos para la investigación cualitativa: Software gratuito y herramientas colaborativas. Opción, 31(5), 453-471. https://www.redalyc.org/articulo.oa?id=310/31045570027

Ivala, E. y Gachago, D. (2012). Social media for enhancing student engagement: The use of Facebook and blogs at a University of Technology. SAJHE, 26(1), 152-166.

Kahu, E. (2013). Framing student engagement in Higher Education. Studies in Higher Education, 38(5), 758-773.

Kuh, G. D., Kinzie, J., Buckley, J. A., Bridges, B. K. y Hayek, J. C. (2007). Piecing together the student success puzzle: Research, propositions, and recommendations. ASHE Higher Education Report, 32(5). https://doi.org/10.1002/aehe.3205

Macchiarola, V., Pizzolitto, A., Pugliese, V. y Muñoz, D. (2020). La enseñanza con modalidad virtual en tiempos del covid19. La mirada de los estudiantes de la Universidad Nacional de Río Cuarto. Revista Contextos de Educación, 28(20), 1-13. http://www2.hum.unrc.edu.ar/ojs/index.php/contextos/article/view/1086/1148

Manzuoli, C., Pineda-Baez, C. y Sanchez, A. V. (2019). Supporting student cognitive and agentic engagement: Students' voices. International Journal of Educational Research, 96, 81-90. https://doi.org/10.1016/j.ijer.2019.06.005

Maroco, J., Maroco, A., Campos, J. y Fredricks, J. (2016). University student's engagement: Development of the University Student Engagement Inventory (USEI). Psicología: Reflexão e Crítica, 29, 21. https://doi.org/10.1186/s41155-016-0042-8

Morán, L. (2020). Educación en tiempos de pandemia: consejos de especialistas para enriquecer las aulas virtuales. CONICET, Humanidades y Ciencias Sociales. https://www.conicet.gov.ar/educacion-en-tiempos-de-pandemia-consejos-deespecialistas-para-enriquecer-las-aulas-virtuales/

Narayan, D. (2005). Measuring empowerment: Cross-disciplinary perspectives. World Bank.

Ordorika, I. (2020). Pandemia y educación superior. Revista de la Educación Superior, 49(194), 1-8. https://doi.org/10.36857/resu.2020.194.1120

Padua, J. (Coord.) (1979). Técnicas de investigación aplicadas a las ciencias sociales. Fondo de Cultura Económica.

Pianta, R., La Paro, K. M., y Hamre, B. K. (2008). Classroom assessment scoring system manual: $K-3$. Brookes.

Pick, S., Sirkin, J., Ortega, I., Osorio, P., Martínez, R., Xocolotxin, U. y Givaudan, M. (2007). Escala para medir agencia personal y empoderamiento (ESAGE). Revista Interamericana de Psicología, 41(3), 295-304.

Rama, C. (2008). Un nuevo escenario en la educación superior en américa latina: la educación virtual. En UNESCO, La educación superior virtual en América Latina y el Caribe (pp. 3951). Asociación Nacional de Universidades e Instituciones de Educación Superior Colección Biblioteca de la Educación Superior. 
Reeve, J. (2013). How students create motivationally supportive learning environments for themselves: The concept of agentic engagement. Journal of Educational Psychology, 105(3), 579-595. https://doi.org/10.1037/a0032690

Reeve, J. y Shin, S. (2020) How teachers can support students' agentic engagement. Theory into Practice, 59(2), 150-161. https://doi.org/10.1080/00405841.2019.1702451

Reeve, J., Cheon, S. y Jang, H. (2020). How and why students make academic progress: Reconceptualizing the student engagement construct to increase its explanatory power.

$\begin{array}{llll}\text { Contemporary Educational 1-12. } & \end{array}$ https://doi.org/10.1016/j.cedpsych.2020.101899

Rigo, D. (2020). Percepciones del estudiantado argentino de nivel superior acerca del compromiso, clima del aula virtual y tendencias a futuro: entre posibilidades y limitaciones en tiempos de pandemia. Innovaciones Educativas, 22, 143-161. https://doi.org/10.22458/ie.v22iEspecial.3132

Rigo, D. y Donolo, D. (2019). Análisis de un modelo integrador del compromiso escolar: relaciones entre variables situacionales, escolares, sociales y personales en alumnos de nivel primario de educación. Propósitos y Representaciones, 7(SPE), 316-316. https://doi.org/10.20511/pyr2019.v7nSPE.316

Rigo, D. y Guarido, G. (2020). Mirar la clase universitaria. Compromiso académico, vínculo con el clima de aula. $V$ Congreso online Internacional sobre la Educación en el Siglo XXI (pp. 130-143). https://www.eumed.net/actas/20/educacion/educacion20.pdf

Ríos, D., Bozzo, N., Marchant, J., y Fernández, P. (2010). Factores que inciden en el clima de aula universitario. Revista Latinoamericana de Estudios Educativos, 40(3-4), 105-126.

Riveiro, J. y Anaya Nieto, D. (2004). Educación a distancia y presencial: Diferencias en los componentes cognitivo y motivacional de estudiantes universitarios. RIED, 7(1/2), 65-75.

Rojas Carvajal, C. (2015). Escuela y empoderamiento. Aportes a la superación de la pobreza desde el ámbito de la educación. Ediciones Uniandes.

Rowland, J. (1997). Questioning empowerment: Working with women in Honduras. Oxfam.

Samman, E. y Santos, M. (2009). Agency and empowerment: A review of concepts, indicators and empirical evidence. Oxford poverty and human development initiative. http://www.ophi.org.uk/wp-content/uploads/OPHI-RP10a.pdf

Sampieri, R., Collado, C. y Lucio, P. (2010). Metodología de la investigación (5ª ed.). Mc Graw Hill.

Sangrá Morer, A. (2006). Educación a distancia, educación presencial y usos de la tecnología: una tríada para el progreso educativo. Edutec. Revista Electrónica de Tecnología Educativa, 15(2), 1-7. https://doi.org/10.21556/edutec.2002.15.541

Schindler, L., Burkholder, G. J., Morad, O. A. y March, C. (2017). Tecnología basada en computadoras y participación de los estudiantes: una revisión crítica de la literatura. International Journal of Educational Technology on Higher Education, 14, 25. https://doi.org/10.1186/s41239-017-0063-0

Shernoff, D. (2013). Optimal learning environments to promote student engagement. Springer.

Sriklaub, K., Wongwanich S. y Wiratchai, N. (2015). Development of the classroom climate measurement model. Procedia - Social and Behavioral Sciences, 171, 1353-1359.

Trowler, V. (2010). Student engagement literature review. The Higher Education Academy. https://pure.hud.ac.uk/en/publications/student-engagement-literature-review 
Uribe-Zapata, A. (2018). Concepto y prácticas de educación expandida: una revisión de la literatura académica. El Ágora USB, 18(1), 277-292.

Veiga, F. (2013). Envolvimento dos alunos na escola: elaboração de uma nova escala de avaliação. INFAD Revista de Psicología, 1(1), 441-450.

Vibert, A. B. y Shields, C. (2003). Approaches to student engagement: Does ideology matter? McGill Journal of Education, 38(2), 221-239.

Wheldall K., Beaman R. y Mok, M. (1999). Does the Individualized Classroom Environment Questionnaire (ICEQ) measure classroom climate? Educational and Psychological Measurement, 59(5), 847-854.

Zhang, Z. y McNamara, O. (2018). Undergraduate student engagement theory and practice in China and the UK. Springer. https://doi.org/10.1007/978-981-13-1721-7 